\title{
Detection of Gravitational Waves from the Coalescence of Population III Remnants with Advanced LIGO
}

\section{Citation}

Wyithe, J. Stuart B., and Abraham Loeb. 2004. “Detection of Gravitational Waves from the Coalescence of Population III Remnants with Advanced LIGO." The Astrophysical Journal 612 (2): 597-601. https://doi.org/10.1086/422183.

\section{Permanent link}

http://nrs.harvard.edu/urn-3:HUL.InstRepos:41393365

\section{Terms of Use}

This article was downloaded from Harvard University's DASH repository, and is made available under the terms and conditions applicable to Other Posted Material, as set forth at http:// nrs.harvard.edu/urn-3:HUL.InstRepos:dash.current.terms-of-use\#LAA

\section{Share Your Story}

The Harvard community has made this article openly available. Please share how this access benefits you. Submit a story. 


\title{
DETECTION OF GRAVITATIONAL WAVES FROM THE COALESCENCE OF POPULATION III REMNANTS WITH ADVANCED LIGO
}

\author{
J. Stuart B. Wyithe ${ }^{1}$ and Abraham Loeb ${ }^{2}$ \\ Received 2003 December 3; accepted 2004 April 12
}

\begin{abstract}
The comoving mass density of massive black hole (MBH) remnants from pregalactic star formation could have been similar in magnitude to the mass density of supermassive black holes (SMBHs) in the present-day universe. We show that the fraction of MBHs that coalesce during the assembly of SMBHs can be extracted from the rate of ring-down gravitational waves that are detectable by the Advanced Laser Interferometer GravitationalWave Observatory (LIGO). Based on the SMBH formation history inferred from the evolution of the quasar luminosity function, we show that an observed event rate of $1 \mathrm{yr}^{-1}$ will constrain the SMBH mass fraction that was contributed by MBH coalescence down to a level of $\sim 10^{-6}$ for $20 M_{\odot} \mathrm{MBH}$ remnants (or $\sim 10^{-4}$ for $260 M_{\odot}$ remnants).
\end{abstract}

Subject headings: black hole physics — cosmology: theory — galaxies: formation

\section{INTRODUCTION}

The first episode of star formation (Population III) occurred in metal-free gas that cooled to low temperatures through collisional excitation of molecular hydrogen, $\mathrm{H}_{2}$ (Bromm \& Larson 2004). Numerical simulations indicate that the first stars were probably more massive than the Sun by 2 orders of magnitude (Bromm et al. 1999; Abel et al. 2000). Population III stars with a mass $M_{*} \gtrsim 260 M_{\odot}$ or $25 \lesssim\left(M_{*} / M_{\odot}\right) \lesssim 140$ end their life by forming a massive black hole $(\mathrm{MBH})$ remnant (Heger \& Woosley 2002; Heger et al. 2003). Madau \& Rees (2001) estimate that the comoving mass density in MBHs at $z \sim 20$ may have been comparable to the observed mass density of supermassive black holes (SMBHs) in the present-day universe. Thus, in principle, the coalescence of MBH remnants could have made a significant contribution to the SMBH mass budget.

Recent semianalytic calculations of the merger history of SMBHs (Islam et al. 2003) find that although the mass density in Population III MBH remnants may be sufficient to provide the present-day density of SMBHs, most of the MBHs end up in satellites and populate galaxy halos. Nevertheless, hierarchical merging inevitably leads to the formation of early dwarf galaxies, which could sink via dynamical friction to make dense clusters of MBHs at the centers of massive galaxies that are assembled later (Madau \& Rees 2001). These central MBH clusters provide sites for MBH coalescence and could produce seeds for SMBH growth (analogous processes take place within the segregated cores of globular star clusters; see Portegies Zwart \& McMillan 2000).

The rise of the SMBH population is traced by the evolution of the quasar luminosity function (e.g., Yu \& Tremaine 2002). While the gas accretion history of the quasar population accounts for most of the locally observed SMBH mass, coalesced MBHs may also contribute some small fraction, $f_{\mathrm{MBH}}$, of that mass. For a given value of $f_{\mathrm{MBH}}$, the coalescence rate

\footnotetext{
${ }^{1}$ University of Melbourne, Parkville, Victoria, Australia; swyithe@isis.ph .unimelb.edu.au.

${ }^{2}$ Harvard-Smithsonian Center for Astrophysics, 60 Garden Street, Cambridge, MA 02138; aloeb@cfa.harvard.edu.
}

of MBHs corresponding to the buildup of mass in SMBHs may then be calculated based on the observed quasar history.

In this paper we show that detection of gravitational waves from MBH coalescences by the Advanced Laser Interferometer Gravitational-Wave Observatory (LIGO II) ${ }^{3}$ will constrain the fraction of the SMBH mass that was assembled through coalescence of MBHs and provide a direct probe of the early population of very massive stars. We use the observed quasar luminosity function to infer that a density $\rho_{\text {acc }}$ of SMBH mass is accreted per unit time at redshift $z$ under the following three assumptions: (1) we assume that at redshift $z$, a density $\rho_{\mathrm{mrg}}=f_{\mathrm{MBH}} \rho_{\text {acc }}$ was formed via coalescence of MBHs during the preceding Hubble time (the total coalescence rate is then determined by integrating over redshift); (2) the coalescence of the population of remnants corresponding to the accreted density at each redshift is assumed to occur over the preceding Hubble time through an evenly spaced coalescence of MBHs; and (3) the value of $f_{\mathrm{MBH}}$ is assumed to be constant with redshift.

\section{RING-DOWN GRAVITATIONAL WAVES FROM MBHs AND DETECTION BY LIGO II}

First, we consider the expected gravitational radiation signal from merging MBHs at high redshifts. The coalescence process of two black holes can be separated into three phases: an inspiral phase, a coalescence phase, and finally a ringing phase of the merged black hole product (Flanagan \& Hughes 1998) as it settles to a Kerr metric. Of these, the inspiral and ringing phases generate well-understood waveforms. Inspiraling MBHs at high redshift will be most luminous at frequencies near $1 \mathrm{~Hz}$, just below the sensitivity band for LIGO II. However, following a merger the MBHs would ring at higher frequencies that are within the LIGO II sensitivity band.

To compute the gravitational wave strain for black hole ringing, we follow the formalism outlined by Hughes (2002). The dimensionless strains for the two polarizations of the

\footnotetext{
3 Additional information on advanced LIGO is available at http://www.ligo caltech.edu/advLIGO.
} 
gravitational waveform during the ring down may be written as

$$
h_{+,-}(t)=A_{+,-} \exp \left(-\pi f_{\text {rd,obs }} t / Q\right) \cos \left(2 \pi f_{\text {rd,obs }} t\right),
$$

where $Q \equiv \pi f_{\text {rd,obs }} \tau_{\text {obs }}$ is the quality factor, $\tau_{\text {obs }}$ is the observed decay time, and $t$ is the observed time following the merger. The observed frequency, $f_{\text {rd.obs }}$, equals the intrinsic frequency, $f_{\text {rd }}$, divided by $(1+z)$. Assuming that the ratio of the strain amplitudes for the two polarizations follows that of the inspiral phase,

$$
A_{+}=A_{\mathrm{rd}}\left[1+(\hat{\boldsymbol{L}} \cdot \hat{\boldsymbol{n}})^{2}\right] ; \quad A_{\times}=-2 A_{\mathrm{rd}}(\hat{\boldsymbol{L}} \cdot \hat{\boldsymbol{n}}),
$$

where $\hat{\boldsymbol{L}} \cdot \hat{\boldsymbol{n}}$ is the dot product of the unit vectors pointing along the binary orbital angular momentum and the line of sight to the source. The overall strain amplitude $A_{\mathrm{rd}}$ is found by requiring that the binary radiates a fraction $\epsilon_{\mathrm{rd}}$ of its energy (e.g., Fryer et al. 2002). If a black hole of mass $\Delta M_{\mathrm{bh}}$ merges with a black hole of mass $M_{\mathrm{bh}}$,

$$
A_{\mathrm{rd}}=10^{-23}\left(\frac{D}{10 \mathrm{Gpc}}\right)^{-1} \sqrt{\frac{20 \epsilon_{\mathrm{rd}}}{4 \pi f_{\mathrm{rd}} Q}\left(\frac{M_{\mathrm{bh}}+\Delta M_{\mathrm{bh}}}{M_{\odot}}\right)},
$$

where $D$ is the comoving distance.

Distortions of the Kerr metric during the ringing phase may be decomposed into spheroidal modes with spherical harmoniclike indices $l$ and $m$ (Fryer et al. 2002). The quadrupole $(l=2)$ moments are expected to dominate the ring-down spectrum. Binary coalescence excites a barlike deformation of the event horizon (Hughes 2002). As a result, the $m=2$ mode dominates over the $m=0$ mode when the ring down forms the final stage of a binary merger. Fits to numerical simulations show that for $l, m=2$ (Leaver 1985; Echeverria 1989; Fryer et al. 2002),

$$
\begin{gathered}
f_{\mathrm{rd}} \sim \frac{10^{5.3} \mathrm{~Hz}}{2 \pi}\left(\frac{M_{\mathrm{bh}}+\Delta M_{\mathrm{bh}}}{M_{\odot}}\right)^{-1}\left[1-0.63(1-a)^{3 / 10}\right], \\
Q=2(1-a)^{-9 / 20},
\end{gathered}
$$

where $a$ is the dimensionless black hole spin, taken to have representative values of $1,0.5$, or 0 in the numerical results presented later. Hughes \& Blandford (2003) have found that equal-mass mergers may have rapidly rotating remnants $(a \sim 1)$, while mass ratios of 0.5 lead to typical spins of $a \sim 0.5$. We note that in addition to setting the oscillation frequency, the spin governs the value of $Q$ and hence the number of oscillations. Ring-down waveforms with more oscillations will be easier to detect.

The observed strain may be written as

$$
H(t)=F_{+}(\theta, \phi, \psi) h_{+}(t)+F_{\times}(\theta, \phi, \psi) h_{\times}(t),
$$

where $F_{+}(\theta, \phi, \psi)$ and $F_{+}(\theta, \phi, \psi)$ are the detector response functions for a source with a sky position $(\theta, \phi)$ and polarization axes rotated at an angle $\psi$ (e.g., Thorne 1987). Since the ring-down waves lie in a narrow frequency range, the signalto-noise ratio $\rho$ for a detection utilizing matched filtering techniques may be evaluated as (Hughes 2002)

$$
\rho^{2}=\frac{2 \int_{0}^{\infty} d t H(t)^{2}}{S_{h}\left(f_{\text {rd }, \text { obs }}\right)}
$$

where $S_{h}\left(f_{\text {rdobs }}\right)$ is the spectral density of detector noise (in $\left.\mathrm{Hz}^{-1}\right)$.

\section{MBH MERGERS AND THE EVOLUTION OF THE SMBH POPULATION}

In the redshift interval between $z_{q}$ and $z_{q}+d z_{q}$, the accretion due to quasars implies that the comoving mass density in SMBHs is

$$
d \rho_{\mathrm{bh}}\left(z_{q}\right)=d z_{q} \int_{0}^{\infty} d L_{B} \frac{L_{\mathrm{bol}}}{\epsilon c^{2}} \Psi\left(L_{B}, z_{q}\right) \frac{d t}{d z_{q}},
$$

where $L_{\text {bol }}$ is the bolometric luminosity of a quasar with a $B$-band luminosity $L_{B}, \epsilon$ is the conversion efficiency of accreted mass to electromagnetic radiation (we adopt $\epsilon=0.1$; Yu \& Tremaine 2002), and $\Psi\left(L_{B}, z_{q}\right)$ is the comoving density of quasars per unit $B$-band luminosity at $z_{q}$, for which we adopt the parametric form derived from the $2 \mathrm{dF}$ survey (Boyle et al. 2000). By integrating equation (8) over redshift, one may obtain the SMBH mass density accreted by a redshift $z_{q}$. The local $\left(z_{q}=0\right)$ value for the comoving mass density in SMBHs accreted during the quasar activity is $\rho_{\text {bh }} \approx 2.7 \times$ $10^{5} M_{\odot} \mathrm{Mpc}^{-3}$. This number is similar to estimates of the local SMBH mass density obtained from the inventory of quiescent galactic SMBHs (e.g., Yu \& Tremaine 2002; Aller \& Richstone 2002).

If some fraction of the SMBH mass is assembled through coalescence during the hierarchical buildup of galaxies, then it is natural to expect MBH remnants to contribute to the SMBH mass budget as they are more massive than normal stellar remnants and should sink to the centers of galaxies by dynamical friction (Madau \& Rees 2001). The initial coalescence of the MBHs would be detectable by Advanced LIGO, and the observed event rate (or lack thereof) could shed light on the possibility that MBH coalescence events produced the early seeds of SMBHs. Suppose that a fraction $f_{\mathrm{MBH}}$ of the mass in SMBHs at any redshift was added through coalescence of MBHs, with the remainder added through gas accretion. Since the comoving mass density of accreted gas mass for quasars is comparable to the final SMBH mass density in the local universe, we expect $f_{\mathrm{MBH}} \ll 1$ independent of other considerations.

While the accreted mass is being added at redshift $z_{q}$, the coalescence of the MBHs incorporated into the SMBH at $z_{q}$ would have stretched over most of the age of the universe $t_{\text {age }}\left(z_{q}\right)$, corresponding to the assembly time of the SMBH. The MBHs are brought together through hierarchical merging of the minihalos that form the building blocks for the massive dark matter halo hosting any particular SMBH. There are several issues to consider, including the number and clustering of MBHs inside minihalos, the merger rate of minihalos into protogalaxies and more massive hosts, and the subsequent dynamical behavior of the MBHs within the resulting gaseous and stellar environments. Rather than model the coalescence history using a dark matter halo merger tree and the poorly understood dynamics of the MBH population, we instead assume that the MBHs undergo random hierarchical coalescences with the MBH mergers distributed linearly in time. At any point in the coalescence history, two MBHs that coalesce are chosen at random from the MBH population. The next merger is then drawn from the revised population, which contains one fewer MBH in total, one additional MBH of a larger mass, and so on. There are a 

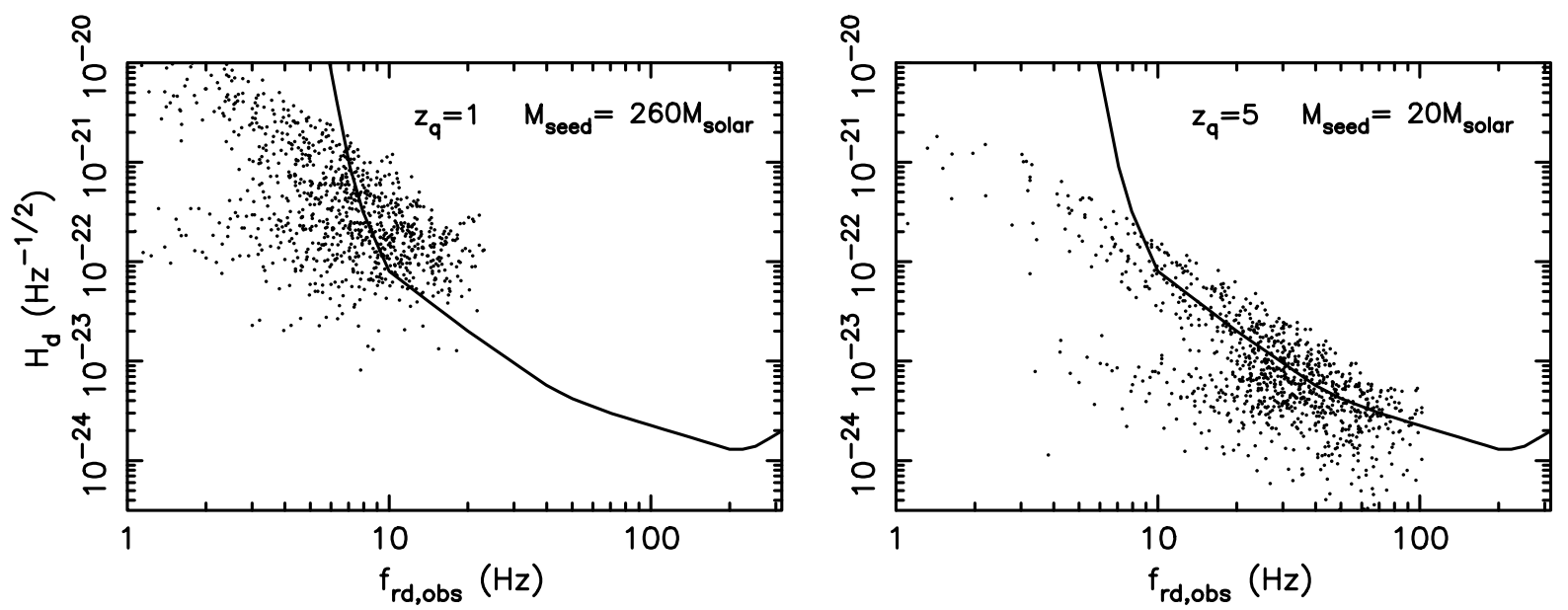

FIG. 1.-Comparison between the ring-down strain signals $H_{d}$ and the LIGO II sensitivity curve as a function of observed frequency, $f_{\text {rd,obs }}$. The points correspond to hierarchical coalescence of $10^{3} \mathrm{MBH}$ seeds to a single MBH at a redshift $z_{q}$. Two cases are shown, having MBH seeds of 20 and $260 M_{\odot}$, coalescing by $z_{q}=5$ and 1 , respectively. Each coalescence event involves a different randomly chosen combination of the parameters $\theta, \phi, \psi, \lambda$, and $\hat{\boldsymbol{L}} \cdot \hat{\boldsymbol{n}}$. The calculations assume $\epsilon_{\mathrm{rd}}=0.03$ and $a=1.0$.

total of $N-1$ coalescence events leading from the $N$ initial seed MBHs to the single final $\mathrm{MBH}$ of mass $N M_{\mathrm{MBH}}$. Onethird of these coalescence events are between MBHs of the initial seed mass.

Figure 1 shows the signal amplitude $H_{d} \equiv\left[2 \int_{0}^{\infty} d t H(t)^{2}\right]^{1 / 2}$ for the hierarchical coalescence of $10^{3} \mathrm{MBH}$ seeds to a single $\mathrm{MBH}$ at a redshift $z_{q}$. Two cases are illustrated, having MBH seeds of 20 and $260 M_{\odot}$ coalescing by $z_{q}=5$ and 1 , respectively. Each individual coalescence event was combined with a different randomly chosen combination of the parameters $\theta$, $\phi, \psi, \lambda$, and $\hat{\boldsymbol{L}} \cdot \hat{\boldsymbol{n}}$. The calculations assume $\epsilon_{\mathrm{rd}} \sim 0.03$ (Hughes 2002) and $a=1.0$. The values of $H_{d}$ in Figure 1 lie along two branches. The lower branch originates from the early stage of the merger tree, where the seed MBHs provide most of the coalescence events. As the typical mass of the coalescing MBHs grows at lower redshifts, the signal amplitude increases while its frequency decreases. The figure also shows $\left[S_{h}\left(f_{\mathrm{rd}, \mathrm{obs}}\right)\right]^{1 / 2}$, the planned sensitivity curve for the two LIGO II $4 \mathrm{~km}$ interferometers (Gustafson et al. 1999). The signal-to-noise ratio $\rho$ is the ratio between the points and the sensitivity curve (see eq. [7]). From an ensemble of such detection histories, we may therefore compute the fraction of mergers $f_{\text {detect }}\left(z_{q}, z_{\text {coal }}\right)$ that are detectable by LIGO II as a function of the quasar redshift, $z_{q}$, and the coalescence redshift, $z_{\text {coal }}$. Note that $f_{\text {detect }}\left(z_{q}, z_{\text {coal }}\right)$ is independent of the initial number of MBH remnants in the tree (for MBHs that are much smaller than the final coalesced SMBH). This is because the fraction of coalescences for MBHs of different masses in the random hierarchical process is independent of the number of MBHs initially in the tree.

The rate at which coalesced MBH mass is added to the SMBH population is given by

$$
\frac{d \rho_{\mathrm{MBH}}}{d z_{q}}=\frac{f_{\mathrm{MBH}}}{1-f_{\mathrm{MBH}}} \frac{d \rho_{\mathrm{bh}}}{d z_{q}},
$$

with the remainder added via gas accretion during the active quasar phase. The number density increment of consumed MBHs can be derived from the mass density increment, $d n_{\mathrm{MBH}}\left(z_{q}\right)=d \rho_{\mathrm{MBH}}\left(z_{q}\right) / M_{\mathrm{MBH}}$.
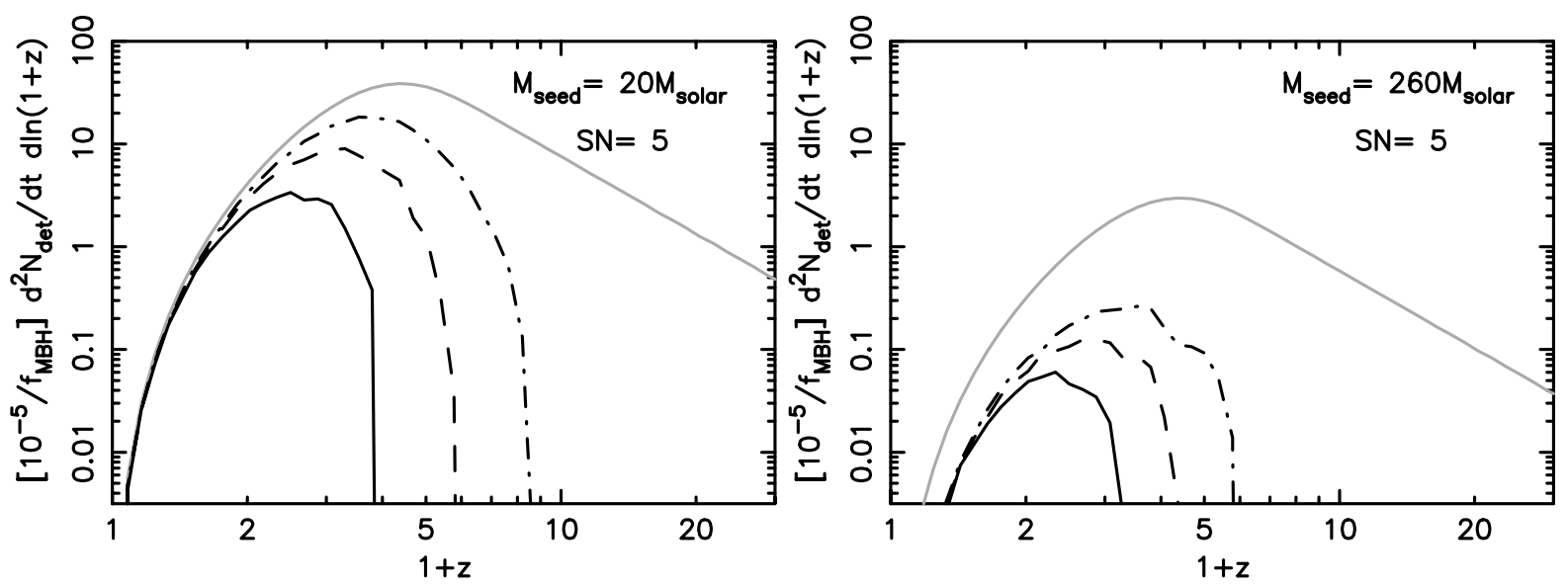

FIG. 2.-Rate of detectable mergers per $\ln (1+z)$ in units of $10^{5} f_{\mathrm{MBH}} \mathrm{yr}^{-1}$. Curves are shown for a signal-to-noise ratio of 5 and initial MBH mass of $20 M_{\odot}$ (left) and $260 M_{\odot}\left(\right.$ right). Each panel shows three curves for $\epsilon_{\mathrm{rd}}=0.01$ (solid curve), 0.03 (dashed curve), and 0.10 (dot-dashed curve). The calculations assumed $a=1.0$. For comparison, we also show the total number of mergers per year in the observer frame (light curves). 
TABLE 1

Detection Rate of Coalescence Events in Units of $10^{5} f_{\mathrm{MBh}} \mathrm{yr}^{-1}$

\begin{tabular}{|c|c|c|c|c|c|}
\hline \multirow{2}{*}{$\begin{array}{l}M_{\text {seed }} \\
\left(M_{\odot}\right)\end{array}$} & \multirow[b]{2}{*}{$a$} & \multirow[b]{2}{*}{$\left(10^{-5} / f_{\mathrm{MBH}}\right)\left(d N_{\mathrm{mrg}} / d t\right)$} & \multicolumn{3}{|c|}{$\left(10^{-5} \mathrm{yr} / f_{\mathrm{MBH}}\right)\left(d N_{\mathrm{det}} / d t\right)$} \\
\hline & & & $\epsilon_{\mathrm{rd}}=0.01$ & $\epsilon_{\mathrm{rd}}=0.03$ & $\epsilon_{\mathrm{rd}}=0.10$ \\
\hline $20,260 \ldots \ldots \ldots \ldots \ldots$ & 1.0 & $35.6,2.7$ & $1.9,0.02$ & $5.7,0.07$ & $13.3,0.15$ \\
\hline $20,260 \ldots \ldots \ldots \ldots \ldots \ldots \ldots$ & 0.5 & $35.6,2.7$ & $1.0,0.003$ & $3.4,0.01$ & $9.8,0.03$ \\
\hline $20,260 \ldots \ldots \ldots \ldots \ldots \ldots$ & 0.0 & $35.6,2.7$ & $0.67,0.001$ & $2.3,0.003$ & $7.5,0.007$ \\
\hline
\end{tabular}

NoтE.-The third column shows the merger rate assuming that all events can be detected.

The redshift distribution of the rate of detectable coalescences (in the observer frame) is

$$
\begin{aligned}
\frac{d^{2} N_{\text {det }}}{d t d z_{\text {coal }}}= & \int_{\infty}^{0} d z_{q}\left[d n_{\mathrm{MBH}} 4 \pi \frac{d V}{d \Omega d z_{q}} \frac{f_{\text {detect }}\left(z_{q}, z_{\text {coal }}\right)}{\left[t_{\text {age }}\left(z_{q}\right)\right]^{2}}\right. \\
& \left.\times \frac{d t}{d z_{\text {coal }}}\left(\frac{1}{1+z_{\text {coal }}}\right) \Theta\left(z_{\text {coal }}-z_{q}\right)\right]
\end{aligned}
$$

where the factor $1 /\left(1+z_{\text {coal }}\right)$ is due to cosmological time dilation, the derivative $d t / d z_{\text {coal }}$ enforces a linear distribution of coalescence events in time, $\left(d V / d \Omega d z_{q}\right)$ is the comoving volume per unit solid angle per unit redshift, and $\Theta(x)$ is the Heaviside step function. Note that $d^{2} N_{\text {det }} / d t d z_{\text {coal }}$ is independent of quantities like the quasar lifetime or number of active quasar phases per SMBH. We only assume that coalescences account for a fraction $f_{\mathrm{MBH}}$ of the SMBH mass added through accretion at redshift $z$.

Figure 2 shows $d^{2} N_{\text {det }} / d t d \ln \left(1+z_{\text {coal }}\right)$ in units of $10^{5} f_{\mathrm{MBH}} \mathrm{yr}^{-1}$. The plotted curves correspond to a signal-tonoise ratio of 5, assuming an MBH initial mass of $20 M_{\odot}$ (left panels) and $260 M_{\odot}$ (right panels). The calculations were performed for values of $a=1.0$ and $\epsilon_{\mathrm{rd}}=0.01,0.03$, and 0.10 . A fraction as small as $f_{\mathrm{MBH}} \sim 10^{-5}$ yields $\sim 0.1-10$ detectable coalescence events at redshifts $2 \lesssim z_{q} \lesssim 5$ (where the quasar population is substantial and the observed frequency does not redshift out of the Advanced LIGO band above $10 \mathrm{~Hz}$ ). For comparison, the light lines show the redshift distribution of the entire coalescence rate $\left(f_{\text {detect }}=1\right)$ in the observer frame, $d^{2} N_{\mathrm{mrg}} / d t d \ln \left(1+z_{\text {coal }}\right)$. We find that Advanced LIGO will be able to detect most of the low-redshift coalescences, while the detectable fraction declines at early cosmic times because of the redshift in frequency.

Values of the total event rate $\left(d N_{\text {det }} / d t\right)$ are presented in Table 1 in units of $10^{5} f_{\mathrm{MBH}} \mathrm{yr}^{-1}$. In addition to the examples plotted in Figure 2, the table lists results for $\mathrm{BH}$ spins of $a=0.0$ and 0.5 . The rates are lower for smaller MBH spins and for larger MBH masses, because of the lower emission frequency and smaller numbers of MBHs.

\section{DISCUSSION}

We have estimated the number of seed MBHs that must have coalesced at high redshifts in order to contribute a fraction $f_{\mathrm{MBH}}$ of the mass density in quasar SMBHs. We then found the corresponding detection rate of ring-down gravitational waves for the Advanced LIGO interferometers. If the initial mass of MBH remnants was $20 M_{\odot}$, then one event per year would imply a coalesced mass fraction of $f_{\mathrm{MBH}} \sim 10^{-6}$. On the other hand, if the MBH seed mass was $260 M_{\odot}$, then one event per year would imply a coalesced mass fraction of $f_{\mathrm{MBH}} \sim 10^{-4}$. Advanced LIGO will be primarily sensitive to MBH coalescence at $z \sim 2-3$, while our calculation implies that most of the MBHs for which coalescence is possible may have coalesced earlier. However, values of $f_{\mathrm{MBH}} \sim 10^{-4}$ to $10^{-6}$ correspond to only a few events per typical galactic nucleus per Hubble time, a very small coalescence rate that is comparable to the rate by which massive galaxies merge.

It is possible that not all MBH coalescences contribute to the assembly of the SMBH mass. In particular, MBHs that are born into tight binaries might coalesce, even if they do not migrate into dense stellar systems or MBH clusters. If $\mathrm{MBH}$ binaries did coalesce without adding mass to the assembled SMBHs, then the observed event rate would only provide an upper limit on $f_{\mathrm{MBH}}$. It is also possible that a fraction of the SMBH mass formed directly as a SMBH from the collapse of a supermassive star (Loeb \& Rasio 1994; Bromm \& Loeb 2003a, 2003b). However, a nonvanishing fraction of direct SMBH formation does not impinge on the estimates of the relative fractions of accreted mass and coalesced MBH remnant mass presented in this paper. Finally, MBHs that coalesced directly with SMBHs without first undergoing coalescence with other MBHs would be invisible to Advanced LIGO, but they might be detected by the planned space mission $\operatorname{LISA}$ (Wyithe \& Loeb 2003). ${ }^{4}$

We thank Scott Hughes and Andrew Melatos for helpful comments. This work was supported in part by NASA grant NAG 5-13292, and by NSF grants AST 00-71019 and AST 02-04514 for A. L.

\footnotetext{
${ }^{4}$ Additional information on LISA is available at http://lisa.jpl.nasa.gov.
}

Abel, T., Bryan, G. L., \& Norman, M. L. 2000, ApJ, 540, 39

Aller, M. C., \& Richstone, D. 2002, AJ, 124, 3035

Boyle, B. J., Shanks, T., Croom, S. M., Smith, R. J., Miller, L., Loaring, N., \& Heymans, C. 2000, MNRAS, 317, 1014

Bromm, V., Coppi, P. S., \& Larson, R. B. 1999, ApJ, 527, L5

\section{REFERENCES}

Bromm, V., \& Larson, R. B. 2004, ARA\&A, in press (astro-ph/0311019)

Bromm, V., \& Loeb, A. 2003a, ApJ, 596, 34 2003b, Nature, 425, 812

Echeverria, F. 1989, Phys. Rev. D, 40, 3194

Flanagan, É. É., \& Hughes, S. A. 1998, Phys. Rev. D, 57, 4535 
Fryer, C. L., Holz, D. E., \& Hughes, S. A. 2002, ApJ, 565, 430

Gustafson, E., Shoemaker, D., Strain, K., \& Weiss, R. 1999, LSC White Paper on Detector Research and Development (LIGO project document T99008000-D; Pasadena: LSC)

Heger, A., Fryer, C. L., Woosley, S. E., Langer, N., \& Hartmann, D. H. 2003, ApJ, 591, 288

Heger, A., \& Woosley, S. E. 2002, ApJ, 567, 532

Hughes, S. A. 2002, MNRAS, 331, 805

Hughes, S. A., \& Blandford, R. D. 2003, ApJ, 585, L101
Islam, R. R., Taylor, J. E., \& Silk, J. 2003, MNRAS, 340, 647

Leaver, E. W. 1985, Proc. R. Soc. London A, 402, 285

Loeb, A., \& Rasio, F. A. 1994, ApJ, 432, 52

Madau, P., \& Rees, M. J. 2001, ApJ, 551, L27

Portegies Zwart, S. F., \& McMillan, S. L. W. 2000, ApJ, 528, L17

Thorne, K. S. 1987, in 300 Years of Gravitation, ed. S. Hawking \& W. Israel (Cambridge: Cambridge Univ. Press), 330

Wyithe, J. S. B., \& Loeb, A. 2003, ApJ, 590, 691

Yu, Q., \& Tremaine, S. 2002, MNRAS, 335, 965 